\title{
ALEXITIMIA Y CARACTERÍSTICAS PSICOLÓGICAS ASOCIADAS A ACTITUDES ALIMENTARIAS EN UNA MUESTRA DE ADOLESCENTES
}

\author{
HIPÓLITO MERINO, AGUSTÍN GODÁS Y M. ${ }^{\circledR}$ GUADALUPE POMBO \\ Universidad Santiago de Compostela
}

(Aceptado en diciembre de 2001)

\begin{abstract}
El objetivo del presente trabajo era examinar la prevalencia de la alexitímica en una muestra no-clínica de sujetos con actitudes alimentarias anómalas (EAT-40 $\geq 20$ ). A los 220 sujetos de la muestra se les administró la Escala de Alexitimia de Toronto (TAS20) y la Escala de Actitudes Alimentarias (EAT-40). A partir de los análisis realizados se observó que los sujetos $(N=39)$ con una tendencia a manifestar síntomas de anore$x i a(E A T \geq 20)$ presentaban puntuaciones en alexitimia superiores a las del grupo control $(N=181)$; concretamente, estos sujetos presentaban una puntuación más elevada en las subescalas afectivas de la TAS-20, a saber, dificultad para identificar los sentimientos y dificultad para describir los sentimientos, pero no presentaban los déficits cognitivos de la alexitimia.
\end{abstract}

Palabras Clave: Trastornos alimentarios, alexitimia, expresión emocional, imagen corporal, adolescencia.

\section{Alexithymia and psychological characteristics associated to eating attitudes in a sample of adolescents}

The aim of this study was to examine the prevalence of alexithymia in a non-clinical sample of subjects with abnormal eating attitudes (EAT $\geq 20)$. Subjects $(N=220)$ completed the Toronto Alexithymia Scale (TAS-20) and the Eating Attitude Test (EAT-40). Results suggest that subjects $(N=39)$ with a tendency to have symptoms of anorexia $(E A T \geq 20)$ showed higher scores on the alexithymia scale than the control group $(N=$ 181); particularly, these subjects shown a higher score on the affective subscales of the TAS-20 (i.e., difficulty identifying feelings and difficulty describing feelings), but not on cognitive deficits of alexithymia.

Key words: Eating disorders, alexithymia, emotional expression, body image, adolescence.

\section{INTRODUCCIÓN}

La "cultura de la delgadez»(Vandereycken y Meerman, 1984) en la que actualmente nos vemos inmersos conduce a una serie de comportamientos y/o actitudes relacionadas con el cuerpo que nos obligan a centrarnos en un trastorno concreto ligado a la adolescencia, la anorexia nerviosa. El progresivo aumento en la

Correspondencia: Hipólito Merino, Departamento de Psicología clínica y Psicología, Facultad de Psicología Campus universitario Sur, 15706 Santiago de Compostela. Correo-e: hmerino@usc.es incidencia de este trastorno dentro de las sociedades desarrolladas ha fomentado la investigación de las posibles causas que lo desencadenan y mantienen.

La literatura en el área de los trastornos alimentarios ha sugerido una relación entre anorexia nerviosa y emociones. Las iniciales teorías psicodinámicas de la anorexia (Bruch, 1973) han atribuido el trastorno a un déficit en la percepción y expresión de las sensaciones corporales internas asociadas a la actividad emocional. Más tarde, los modelos cognitivos (Herman y Polivy, 1980) 
describieron el efecto perturbador que las emociones fuertes ejercían en los individuos sometidos a una restricción alimentaria, así como también se ha destacado el papel de las creencias o pensamientos negativos relacionados con el peso y la forma corporal como responsables directos del trastorno anoréxico (Vitousek y Orimoto, 1993). Estos postulados teóricos han conducido a clínicos e investigadores al estudio de la experiencia emocional en los individuos que lo padecen y más concretamente el papel de la alexitimia en este tipo de trastornos. Parece que en la anorexia nerviosa se observa, entre sus características, la presencia de alexitimia, al menos en lo que a dificultad para identificar y describir los sentimientos se refiere.

Aunque inicialmente el concepto de alexitimia (Sifneos, 1972) surgió ligado a los trastornos psicosomáticos (Sifneos, 1973; Keltikangas-Jarvinen, 1986, 1987; Sriran, Ghatuverdi, Gopinath y Shanmugan, 1987) a medida que aumentaba el número de investigaciones en torno al constructo, se observó que las características alexitímicas estaban presentes en una amplia variedad de trastornos, entre los que se incluyen: trastornos de dolor crónico (Postpone, 1986; Catchlove, Cohen, Braha y Demers-desrosiers, 1985; Acklin y Alexander, 1988; Cox et al.,1994), trastorno por abuso de sustancias (Haviland, Shaw, MacMurray y Cummings, 1988; Rybakowski, Ziolkowski, Zasadska y Brzezinski, 1988; Taylor, Parker y Bagby, 1990) y trastorno de estrés postraumático (Krystal, 1971; Krystal, 1977; Krystal, Giller y Cicchetti, 1986) pero sobre todo se observó una elevada incidencia alexitímica en pacientes con trastorno alimentario (Bourke, Taylor y Crisp, 1985; Bourke, Taylor, Parker y Bagby, 1992; Cochrane, Brewerton, Wilson y Hodges, 1993; Schmidt, Jimany y
Treasure, 1993; Taylor, Parker, Bagby y Bourke, 1996; Laquatra y Clopton, 1994.). En la actualidad, la alexitimia hace referencia a un concepto multidimensional caracterizado por un estilo comunicativo concreto, cuyas características más significativas y destacadas según Taylor et al. (1988) son: (1) dificultad para identificar los sentimientos y diferenciarlos de las sensaciones corporales que acompañan a la actividad emocional (DIS); (2) dificultad para describir los sentimientos propios a los demás (DDS); y (3) patrón de pensamientos concreto o pensamiento orientado externamente (POE), caracterizado por un estilo cognitivo concreto y basado en la realidad.

A pesar de las múltiples investigaciones realizadas sobre la anorexia nerviosa, centradas en el estudio de las características biológicas, conductuales y sociodemográficas, el estudio de la experiencia emocional en los sujetos anoréxicos es todavía limitado. Como un intento de profundizar en el estudio de esta correspondencia establecida entre anorexia y emociones se ha comenzado a investigar la relación entre anorexia nerviosa y alexitimia (Bourke et al.,1985; Bourke et al. 1992; Cochrane et al.1993; Schmidt et al. 1993; Taylor et al. 1996; Laquatra et al. 1994). Aunque de momento son pocos los trabajos llevados a cabo con el propósito de examinar directamente esta relación, los resultados de este grupo de investigaciones coinciden a la hora de señalar que las personas que presentan o padecen algún tipo de trastorno alimentario acusan más esta característica, son más alexitímicos que aquellos que pertenecen al grupo control, concretamente presentan una mayor dificultad para identificar y describir lo que sienten. Estos datos corroboran y confirman la relación establecida entre anorexia y alexitimia, lo que nos lleva 
a pensar en la alexitimia como un rasgo de personalidad estable en los pacientes anoréxicos.

No obstante, a pesar de la preocupación general por la apariencia física y el peso, así como el progresivo aumento de los problemas alimentarios dentro de la población adolescente, sobre todo entre la población femenina, el número de trabajos destinados a examinar la relación entre los comportamientos o actitudes alimentarias y las características alexitímicas en muestras no clínicas es todavía más reducido (Taylor et al. 1996; Laquatra et al.1994). Como consecuencia, en este estudio se ha empleado una muestra no-clínica de jóvenes estudiantes con el objetivo de examinar las actitudes alimentarias de un grupo de adolescentes, por ser ésta la etapa de máximo riesgo para el desarrollo de la patología alimentaria y la relación que éstas guardan con la alexitimia.

De este modo, si se confirma la relación entre los factores o características que predisponen al trastorno alimentario y las características alexitímicas en una muestra de adolescentes, podríamos hipotetizar que la alexitimia constituye un rasgo de personalidad característico de los sujetos que presentan algún problema alimentario, siendo su importancia considerable tanto a nivel etiológico como terapéutico.

\section{MÉTODO}

\section{Participantes}

La muestra estaba formada por 220 estudiantes (102 varones y 118 mujeres) que atendían el Curso de Orientación Universitaria (C. O. U.) en dos Institutos de la provincia de La Coruña. A partir de la puntuación de corte del EAT-40 $(\geq 20)$ se establecieron dos grupos: (1) sujetos con tendencia a padecer la patología alimentaria y (2) grupo control.

\section{Procedimiento y pruebas psicológicas}

Las pruebas se aplicaron en grupos de 2530 sujetos. Al comienzo de cada sesión se dio a cada grupo una breve descripción sobre los objetivos del trabajo y una vez entregados los cuestionarios se les instó a que leyesen detenidamente las instrucciones para posteriormente pasar a rellenar los formularios. Se establecieron dos grupos en función de las puntuaciones obtenidas en el Eating Attitude Test (EAT-40), un grupo con tendencia a patología alimentaria y un grupo de control. Se aplicaron las siguientes pruebas psicológicas:

Eating Attitude Test (EAT-40; Garner y Garfinkel, 1979). Es un cuestionario diseñado para registrar la presencia de características anoréxicas, especialmente las relacionadas con el miedo a ganar peso, el impulso de adelgazar y la presencia de patrones alimentarios restrictivos. Consta de 40 items, cada uno de los cuales se valora en una escala de tipo Likert de 6 puntos. En la actualidad, se dispone de datos obtenidos con muestras españolas (Toro et al. 1989). Así como también resultados sobre la validación de la versión castellana de la misma llevada a cabo en una muestra de 78 pacientes con anorexia y un grupo control de igual tamaño. Se estableció un coeficiente alpha de fiabilidad de 0,61 $(p<0,01)$. El estudio de la validez discriminante aconseja establecer la puntuación criterio en $\geq 20$ a fin de obtener una sensibilidad del $91 \%$ y una especificidad del $69.2 \%$. En el análisis factorial de la escala se obtuvieron tres factores que explicaban el $41 \%$ de la varianza total. La fiabilidad de la escala para el grupo clínico y el control juntos fue de 0,93 y para el grupo clínico aislado fue de 0,92. 
Twenty-Item Toronto Alexithymia Scale (TAS-20; Bagby, Parker y Taylor, 1994). Un cuestionario de autoinforme compuesto de tres factores, que miden la dificultad para identificar los sentimientos (Factor 1), dificultad para comunicar los sentimientos propios a los demás (Factor 2) y pensamiento orientado externamente (Factor 3). Estos factores explicaban un $27.9 \%$ de la varianza total y comprenden un conjunto de items congruentes con la definición teórica del constructo. La puntuación total de la escala oscila entre un rango comprendido entre 20 y 100 . Cada uno de los items se valora en función de una escala Likert que va desde 1 (nada de acuerdo) hasta 5 (totalmente de acuerdo).La puntuación de corte universalmente aceptada es $\geq$ 61( Sandín y Chorot, 1995). La TAS-20 ha mostrado una fiabilidad (.81) y validez apropiadas.

Cuestionario de Imagen Corporal. Hemos diseñado un cuestionario personal para recoger información sociodemográfica e información sobre el peso, alimentación y percepción corporal propia. Las preguntas del cuestionario de imagen corporal eran: (1) Con tu peso actual, ¿te sientes? (2) Con tu peso actual, ¿cómo te perciben los demás? (3) ¿Hasta qué punto te han puesto en ridículo a causa de tu cuerpo? (4) Actualmente, ¿cuál es tu grado de satisfacción con tu cuerpo? (5) Actualmente, ¿cuál es tu grado de satisfacción con tu apariencia física? (6) ¿Te has sometido alguna vez a una dieta estricta para adelgazar? (7) ¿Con qué frecuencia te sometes a dietas estrictas para adelgazar? (8) ¿Alguno de tus familiares o amigos más íntimos hacen dieta para adelgazar? (9) ¿Cómo te gustaría ser?

Índice de Masa Corporal (IMC; Body Mass Index, BMI). A partir de la altura al cuadrado, expresada en metros, y del peso expresado en kilogramos calculamos el Índice de Masa Corporal para cada individuo. [IMC de Quetelet = $\mathrm{kg} /$ metros $^{2}$ (Beumont et al., 1988)]. Normalmente se suele considerar que un IMC de 30 o más es indicativo de obesidad, mientras que un valor inferior a 18 lo es de bajo peso grave, siendo un índice de masa corporal comprendido entre 20-25 indicativo de Peso Normal.

\section{RESULTADOS}

\section{Características generales de la muestra}

El total de la muestra estaba formada por 102 varones $(46,36 \%)$ y 118 mujeres $(53,64 \%)$, lo que supone una proporción ligeramente superior de mujeres que de varones. La edad media de la muestra fue de 17,7 años (DT $=0,89$ ), y el $95,9 \%$ del total tenía una edad comprendida entre los 16 y los 19 años. Más de la mitad del total de la muestra presentaba unos ingresos familiares medios y el $65 \%$ residía en una zona rural. Conforme al IMC se observó que algo más de la mitad de la muestra $(61,4 \%)$ presentaba peso normal y el $27,8 \%$ presentaba bajo peso.

A partir del cuestionario de imagen corporal observamos que aunque no más del $10,9 \%$ de la muestra presenta un peso superior al considerado como normal, un $35 \%$ se percibía a sí mismo como gordo y en torno al $19 \%$ afirmaba sentirse insatisfecho con su cuerpo y apariencia externa. Además, un $9,5 \%$ se había sometido en algún momento de su vida a una dieta restrictiva con el objetivo de perder peso y en el momento de la evaluación el $1,8 \%$ estaba sometido a una dieta diaria. No es de extrañar, pues, que alrededor del $39 \%$ del total de la muestra presentase un fuerte deseo de adelgazar. Estos porcentajes son superiores si nos centramos exclusivamente en la población de mujeres. 
En cuanto a los datos relativos a los cuestionarios psicológicos (EAT y TAS; véase la Tabla 1) observamos que sólo un $7,27 \%$ del total de la muestra alcanzó o superó la puntuación criterio de $\geq$ 61 en la TAS-20. Para el EAT-40, el porcentaje de personas que alcanzó la puntuación criterio fue del 20,89\%. Dividiendo a la muestra en función de la variable sexo observamos que estas medias se incrementan en el caso de las mujeres para la TAS total y para sus subescalas afectivas (DIS y DDS), así como en el EAT-40.

\section{Analisis diferenciales (análisis de varianza)}

Pese a que se observaron puntuaciones superiores en la TAS y en sus subescalas afectivas entre la población femenina, sólo se observaron diferencias significativas con respecto a la subescala de POE. Los varones presentan un pensamiento más concreto centrado en los detalles y acontecimientos externos $[F(1,218)$ $=3,94 ; \mathrm{p}<0,001$ ] ( ver Tabla 1).

Con respecto al EAT, las chicas presentan una mayor predisposición a padecer la patología alimentaria que los varones y obtienen puntuaciones en el EAT-40 significativamente superiores a las observadas en los varones $[F(1,218)$ $=4,22 ; \mathrm{p}<0,001$ ] (Tabla 1).

No se observaron diferencias significativas en las puntuaciones del EAT-40 ni en las puntuaciones de la TAS-20 o en sus subescalas en función de la variable procedencia. Se observó, curiosamente, que los individuos con ingresos económicos familiares más bajos (100.000 Ptas. o menos) [Media $=17,65$, (DT $=10,79)]$ obtenían puntuaciones en el EAT-40 significativamente superiores a las de los sujetos con ingresos medios o bajos [Media $=12,32(\mathrm{DT}=8,78) \mathrm{y}$ $14,9(\mathrm{DT}=11,33)$, respectivamente] $[\mathrm{F}(1,218)=3.45 ; \mathrm{p}<.05]$.

Los diferentes grupos establecidos a partir del Índice de Masa Corporal parecen determinar las puntuaciones obtenidas en la TAS, concretamente en el POE. Los participantes que presentan obesidad moderada obtenían puntuaciones en el POE significativamente superiores a las observadas en el resto de los grupos $[F(3,216)=3,94, p<0,009]$. Cuando dividimos la muestra en función de la variable sexo, observamos que los varones con sobrepeso presentan una mayor DIS $[F(3,98)=5,37 ; p<0,002]$, así como

Tabla 1. Medidas y desviaciones típicas (DT) para las variables de los cuestionarios TAS-20 y EAT-40

\begin{tabular}{lrrrrrr}
\hline & TAS & DIS & DDS & POE & EAT & IMC \\
\hline Varones $(n=102)$ & & & & & & \\
Media & 48,67 & 13,11 & 12,82 & $20,71^{\star *}$ & 11,37 & 22,82 \\
DT & 9,57 & 4,86 & 3,93 & 4,51 & 5,52 & 2,44 \\
\hline Mujeres $(n=118)$ & & & & & & \\
Media & 49,05 & 14,32 & 13,47 & 19,14 & $15,86^{\star \star}$ & 21,17 \\
DT & 10,35 & 4,86 & 4,70 & 4,43 & 12,36 & 2,35 \\
\hline Grupo total $(n=220)$ & & & & & & \\
Media & 48,87 & 13,76 & 13,17 & 19,87 & 13,78 & 21,94 \\
DT & 9,98 & 4,89 & 4,36 & 4,53 & 10,04 & 2,52 \\
\hline Nota
\end{tabular}

Nota $:$ TAS = Toronto Alexithymia Scale; DIS = dificultad para identificar los sentimientos; DDS = dificultad para describir los sentimientos; POE = pensamiento orientado externamente; $E A T$ = eating attitude test; IMC: índice de masa corporal. 
una puntuación en el EAT significativamente superior $[F(3,98)=4.22 ; \mathrm{p}<$ 0,008 ]. No se observaron diferencias significativas en las chicas en función de su IMC ni en la TAS-20 ni en los demás cuestionarios incluidos en el estudio (véase la Tabla 2).

Por lo que respecta a los items del Cuestionario de Imagen Corporal, observamos que con respecto a cómo se siente la persona con su peso actual, aquellos individuos que se autoperciben delgados obtienen puntuaciones superiores en el POE $[F(6,213)=2,64 ; p<$ $0,017]$ y en el EAT $[F(6,213)=17,98 ; p$ $<0,000$ ). Por otra parte, los sujetos que se autoperciben como gordos son los que presentan un mayor IMC $[F(6,213)$ $=11,30 ; p<0,000$ ).
En cuanto al ítem « Con tu peso actual, ¿Cómo te perciben los demás?» observamos que aquellos sujetos que creen que los demás los perciben como extremadamente gordos o extremadamente delgados, obtienen puntuaciones elevadas en la subescala de DIS $[F(6,213)=2,29$; $p<0,000]$. Aquellos individuos que dicen creer que los demás los perciben como «gordos», obtienen puntuaciones superiores en el EAT $[F(6,213)=5,13 ; p$ $<0,000]$ y presentan un IMC superior al de aquellos individuos que creen que los demás los perciben «delgados» $[F(6,213)$ $=21.14 ; \mathrm{P}<.000]$.

Las puntuaciones de la DIS $[F(4,215)=$ $3,06 ; p<0,018]$ y del EAT $[F(4,215)=$ $9,91 ; p<0,000]$ son superiores para aquellos individuos que afirman haber sido

Tabla 2. Diferencias en función del Índice de Masa Corporal. Medias (DT entre paréntesis) y valores de $F$ (sólo los significativos estadísticamente)

\begin{tabular}{|c|c|c|c|c|c|}
\hline & Peso bajo & Peso normal & Obesidad moderada & Sobrepeso & $F$ \\
\hline \multicolumn{6}{|l|}{ TAS: } \\
\hline Varones & $49,89(6,70)$ & $47,20(9,17)$ & $52,80(11,17)$ & $57(7,07)$ & \\
\hline Mujeres & $49,17(9,83)$ & $48,96(10,65)$ & $53,33(5,57)$ & $25(2,83)$ & \\
\hline Total & $49,30(9,29)$ & $48,06(9,93)$ & $52,92(10,01)$ & $46,33(19,14)$ & \\
\hline \multicolumn{6}{|l|}{ DIS: } \\
\hline Varones & $13,33(3,12)$ & $12,21(4,22)$ & $15,42(6,08)$ & $22,5(6,36)$ & $5,37^{*}$ \\
\hline Mujeres & $14,44(4,85)$ & $14,29(4,96)$ & $15,17(3,66)$ & $7(1,41)$ & \\
\hline Total & $14,24(4,58)$ & $13,23(4,70)$ & $15,36(5,52)$ & $17,33(10,02)$ & \\
\hline \multicolumn{6}{|l|}{ DDS: } \\
\hline Varones & $13,78(3,99)$ & $12,43(3,84)$ & $13,32(4,06)$ & $18(2,83)$ & \\
\hline Mujeres & $13,59(4,99)$ & $13,5(4,69)$ & $13,67(1,86)$ & $6(2,32)$ & \\
\hline Total & $13,62(4,79)$ & $12,96(4,30)$ & $13,40(3,62)$ & $14(7,21)$ & \\
\hline \multicolumn{6}{|l|}{ POE: } \\
\hline Varones & $20,44(4,77)$ & $20,79(4,41)$ & $21,21(4,26)$ & $14(8,48)$ & \\
\hline Mujeres & $19,05(4,14)$ & $19(4,49)$ & $22,83(3,66)$ & $11(3,53)$ & \\
\hline Total & $19,30(4,24)$ & $19,91(4,52)$ & $21,60(4,11)$ & $13(6,25)$ & $3,94^{*}$ \\
\hline \multicolumn{6}{|l|}{ EAT: } \\
\hline Varones & $10,67(3,67)$ & $10,43(4,62)$ & $14,58(7,57)$ & $18(8,49)$ & $4,22^{\star}$ \\
\hline Mujeres & $13,59(9,16)$ & $16,01(13,47)$ & $27,33(12,86)$ & $29(4,24)$ & \\
\hline Total & $13,06(8,49)$ & $13,18(10,36)$ & $17,64(10,41)$ & $21,67(8,74)$ & \\
\hline
\end{tabular}

Nota : TAS = Toronto Alexithymia Scale; DIS = dificultad para identificar los sentimientos; DDS = dificultad para describir los sentimientos; POE = pensamiento orientado externamente; EAT = eating attitude test; IMC: índice de masa corporal.

${ }^{\star} p<0,01$ 
puestos en ridículo mucho o extremadamente. Con respecto al POE $[F(4,215)=$ 4,$40 ; p<0,002$ ], las puntuaciones en esta escala decrecen a medida que la persona cree no haber sido puesto en ridículo a lo largo de su vida.

Por lo que se refiere a la satisfacción con la imagen corporal y con la apariencia física, observamos que cuanto más insatisfecho está un individuo con estas variables, tanto mayor es su puntuación en el $\operatorname{EAT}[F(4,215)=8.92(p<0,000)$ y $4.67(p<0,001)$, respectivamente].

La variable "dieta" indica que si un individuo se ha sometido a una dieta restrictiva para perder peso en algún momento de su vida. Cuanto mayor es la puntuación en la variable dieta, mayor es el valor en las variables DIS $[F(1,218)=$ $5,64 ; p<0,018]$, DDS $[F(1,218)=4,13 ; p<$ $0,043]$, EAT $[F(1,218)=61,51 ; p<0,000]$ e IMC $[F(1,218)=10,58 ; p<0001]$. De igual modo, cuanto mayor es la frecuencia de dietas, mayor es su puntuación en el EAT $[F(3,216)=2,03 ; p<0,000]$.

Por último, aquellos individuos que deseaban estar más delgados puntuaban más alto en el POE $[F(3,216)=2,90 ; p<$ $0,036]$ y su IMC $[F(3,216)=13,63 ; p<$ $0,000]$ era mayor, mientras que aquéllos que se gustaban o estaban satisfechos tal y como eran (referente al peso) obtenían mayores puntuaciones en el EAT $[F(3,216)=6,17 ; p<0,000]$.

\section{Análisis correlacionales}

Se llevaron a cabo análisis correlacionales entre la TAS, el EAT-40 y los items incluidos en el cuestionario de Imagen Corporal. La puntuación criterio del EAT40 establecida por Castro et al (1991) nos ha servido para dividir a la muestra en dos grupos: 1) sujetos con tendencia a padecer la patología alimentaria (EAT $\geq$ $20 ; n=46)$ y 2) grupo control (EAT $<20$; $n=174$ ). A pesar de la tendencia obser- vada por parte del primer grupo a obtener una puntuación alexitímica superior a la observada en el segundo grupo, concretamente a presentar una mayor dificultad para identificar y describir los sentimientos, no se observaron diferencias significativas entre el EAT-40 y la TAS20. Tampoco se observaron correlaciones significativas entre la TAS-20 y el cuestionario de imagen corporal. Se observó, sin embargo, que el EAT $\geq 20$ correlacionaba negativamente con la autopercepción corporal $(\mathrm{r}=-0,46, p<0,01)$ y el haberse sometido a una dieta restrictiva en algún momento de la vida $(r=-0,58, p$ $<0,001)$, de modo que cuanto mayor fuese la predisposición de un sujeto a presentar o padecer la patología alimentaria, tanto mayor sería su tendencia a percibirse gordo y la probabilidad de someterse a dieta. Asimismo, el EAT $\geq 20$ correlacionaba positivamente con la satisfacción corporal $(\mathrm{r}=0,38, p<0,01) \mathrm{y}$ con la frecuencia de $\operatorname{dietas}(\mathrm{r}=0,61$, $p<0,001$ ), siendo por lo tanto mayor la insatisfacción con la apariencia corporal y la frecuencia de dietas cuanto mayor es la puntuación en el EAT.

\section{DISCUSIÓN}

La mayoría de los trabajos llevados a cabo hasta el momento tanto con muestras clínicas como con muestras no-clínicas han puesto de manifiesto que los sujetos que presentan algún tipo de trastorno alimentario (anorexia y / o bulimia nerviosa) son más alexitímicos que los sujetos incluidos dentro de un grupo control normal. Concretamente, estos individuos con problemas alimentarios tienen más dificultades para identificar y describir lo que sienten, pero no presentan el estilo de pensamiento concreto característico de la alexitimia. Autores como Taylor et al. (1996) han atribuido estos déficits afectivos de la alexitimia 
observados en pacientes con anorexia o bulimia nerviosa a algún déficit o problema en el procesamiento cognitivo emocional que nada tiene que ver con el pensamiento operatorio característico de la misma.

Pese a la evidencia positiva a favor de la relación entre alexitimia y trastornos de alimentación, en el presente trabajo no se han observado correlaciones entre la escala de actitudes alimentarias (EAT-40) y la escala de alexitimia (TAS-20) o sus factores (DIS, DDS, POE). Aunque sí hemos observado una tendencia por parte del grupo de individuos con una puntuación en el EAT $\geq 20$ a obtener una puntuación en la TAS superior, estas diferencias no alcanzaron el nivel de significación estadística.

Sin embargo, este no es el primer trabajo en el que no se observan relaciones entre la alexitimia y características conductuales de los trastornos de alimentación. Schmidt et al. (1993) y Cochrane et al. (1993), observaron que aunque los sujetos diagnosticados con anorexia o bulimia nerviosa presentaban puntuaciones significativamente superiores a las observadas en el grupo control, tampoco obtuvieron correlaciones entre la alexitimia y los comportamientos o actitudes alimentarias (atracones, dieta, etc.). De acuerdo con un trabajo publicado por Taylor et al. (1996), la alexitimia correlaciona positivamente con las características cognitivas o psicológicas de pacientes que presentan o padecen algún tipo de trastorno alimentario, pero no correlacionaría con las actitudes o comportamientos de alimentación (Por ejemplo, peso, dieta, apariencia física). Esta afirmación se ve apoyada por los resultados de nuestro trabajo en el cual no se observaron relaciones entre la alexitimia y ninguno de los items incluidos dentro del cuestionario de imagen corporal. Una posible explicación a este fenómeno pudiera ser que el EAT-40 mide princi- palmente comportamientos o conductas alimentarias y por lo tanto es difícil establecer algún tipo de relación entre alexitimia y patología alimentaria con esta escala. Rastam et al. (1997) observaron que sólo un mínimo porcentaje de sujetos con anorexia nerviosa puntuaban alto en la escala de alexitimia, lo que sugiere que sóo un grupo concreto de sujetos con anorexia, probablemente aquellos que solicitaban tratamiento, presentarían las características afectivas de la anorexia nerviosa. Asimismo, , puesto que estamos trabajando con muestra no-clínica, pudiera suceder que el grupo con tendencia a padecer la patología alimentaria no presentase la suficiente severidad como para mostrar alguna de las características alexitímicas.

La relación establecida entre IMC, EAT y DIS en varones obesos sugiere que éstos se preocupan por su cuerpo, presentando un riesgo a padecer la patología alimentaria, con la consiguiente dificultad para identificar lo que sienten. El hecho de no haber observado relaciones entre la TAS y el IMC en la muestra general y de que la única relación observada se estableciese con un índice de masa corporal elevado en los varones, sugiere que la alexitimia no sería el resultado de la pérdida de peso. No obstante debido a la naturaleza transversal del trabajo y al hecho de utilizar una muestra no-clínica, hace difícil poder establecer si el bajo peso o el sobrepeso constituyen un factor de empeoramiento en el funcionamiento cognitivo de este grupo de sujetos.

Resumiendo, los resultados de este trabajo ponen de manifiesto, que la alexitimia no está presente en adolescentes con riesgo de padecer la patología alimentaria (identificados con el EAT-40). Con lo cual se podría establecer que la alexitimia podría aparecer con el deterioro físico, cognitivo y personal que conlleva la patología alimentaria, pero no estaría presente al inicio de la mis- 
ma. De todos modos, es necesario llevar a cabo estudios longitudinales para aclarar el efecto de la alexitmia en este tipo de trastornos.

Teóricamente, la alexitimia tendría importantes implicaciones en el pronóstico y respuesta al tratamiento en los trastornos alimentarios, pero todavía queda por demostrar si la alexitimia es un rasgo o un estado de la personalidad en individuos con un trastorno alimentario.

\section{REFERENCIAS BIBLIOGRÁFICAS}

Acklin, M.W., y Alexander, G. (1988). Alexithymia and somatitation: A Rorschach study of four psychosomatic groups. The Journal of Nervous and Mental Disease, 176, 343-350.

Bagby, R. M., Taylor, G.J., y Parker J. D. (1994). The Twenty-Item Toronto Alexithymia Scale-I: Item selection and crossvalidation of the factor structure. Journal of Psychosomatic Research, 38, 23-32.

Beaumont, P., AL-Alami, M, y Touyz, S. (1988). Relevance of standard measurement of undernutrition to the diagnosis of anorexia nervosa: Use of the Quetelet's body mass index (BMI). International Journal of Eating Disorders, 7, 399-405.

Bourke, M. P., Taylor, G., y Crisp, A. M. (1985). Symbolic functioning in anorexia nervosa. Journal of Psychiatry Research, 19, 273-278.

Bourke, M.P., Taylor, G.J., Parker, D.A., y Bagby, R.M. (1992). Alexithymia in women with Anorexia Nervosa: A preliminary Investigation. British Journal of Psychiatry, 161, 240-243.

Bruch, H. (1962). Perceptual and conceptual disturbances in anorexia nervosa. Psychosomatic Medicine, 24, 187-194.

Bruch, H. (1973)._Eating Disorders: obesity, anorexia, and the person within. New York: Basic Books.

Bruch, H. (1982). Anorexia Nervosa: Therapy and Theory. The American Journal of Psychiatry, 138, 1531-1538.
Bruch, H. (1990). Anorexia Nervosa. New York: Hoeber.

Catchlove, R. F., Cohen, K..R., Braha, R.E., y Demers-Deesrosiers, L.A. (1985). Incidence and implications of Alexithymia in chronic pain patients. The Journal of Nervous and Mental Disease, 173, 246-248.

Cochrane, C.E., Brewerton, T.D., Wilson, D.B., y Hodges, E.L. (1993). Alexithymia in the Eating Disorders. International Journal of Eating Disorders, 14, 219-222.

Cox, B.J., Kuch, K., Parker, J.D.A., Shulman, I.D., y Evans, R.I. (1994). Alexithymia in somatoform disorder Patients with chronic pain. Journal of Psychosomatic Research, 38, 523-527.

Garner, D.M. y Garfinkel, P.E. (1979). The Eating Attitude Test: And index of the symptoms of anorexia. Psychological Medicine, 10, 273-279.

Haviland, M.G., Shaw, D.G., MacMurray, J.P. y Cummings, M. A. (1988). Validation of The Toronto Alexithymia Scale with substance Abusers. Psychotherapy and Psychosomatics, 50, 81-87.

Herman, C.P. y Polivy, J. (1980). Restrained Eating. En B. Stunkand (Eds.), Obesity. Philadelphia: Saunders.

Hsu, L.K.G., y Sobkiewicz, T.A. (1991). Body Image Disturbance: Time to Abandon the Concept for Eating Disorders?.International Journal of Eating Disorders, 10, 15-30.

Keltikangas-Jarvinen, L. (1986). Concept of Alexithymia: I. The prevalence of Alexithymia in Psychosomatic patients.Psychotherapy and Psychosomatics, 44, 132-138.

Keltikangas-Jarvinen, L. (1987). Concept of Alexithymia: II. The consistency of Alexithymia. Psychotherapy and Psychosomatics, 47, 113-120.

Krystal, H. (1971). Trauma: consideration of severity and chronicity. In $\mathrm{H}$. Krystal y W. Niederland (Eds.), Psychic Traumatization (pp. 11-28). Boston: Little Brown.

Krystal, H. (1977). Self-representation and the capacity for selfcare. Annals of Psychoanalysis, 6, 207-246.

Krystal, H. , Giller, E.L. y Cicchetti, D.V. (1986). Assesment of alexithymia in posttraumatic stress disorder and somatic illness: Introduction of a reliable measure. Psychosomatic Medicine, 48, 84-94. 
Laquatra, T.A. y Clopton, J.R. (1994). Characteristics of Alexithymia and eating disorders in college women. Addictive Behaviors, 19, 373-380.

Postpone, N. (1986). Alexithymia in chronic pain patients. General Hospital Psychiatry, 8, 163-167.

Raich, M. (1994). Anorexia y Bulímia: Trastornos Alimemtarios. Madrid: Pirámide.

Rastam, M., Gillberg, C., Gillberg, I.C. y Johansson, M. (1997). Alexithymia in Anorexia Nervosa: A controlled study using the 20-Item Toronto Alexithymia Scale. Acta Psychiatrica Scadinavica, 5, 385-388.

Rybakowsky, J., Ziolkowski, M., Zasadzka, t. y Brzezinski, R. (1988). High Prevalence of Alexithymia in male patients with alcohol dependence. Drug and Alcohol Dependence, 21, 133-136.

Sandín, B. y Chorot, P. (1995). Escala de Alexitimia de Toronto TAS-20. UNED, Madrid (no publicado).

Schmidt, U., Jimany, A. y Treasure, J. (1993). A controlled study of Alexithymia in eating disorders. Comprehensive Psychiatry, 34, 54-58.

Sifneos, P.E.(1972). Short-term psychotherapy and emotional crisis. Cambridge: Harvard University Press.
Sriran, T.G., Ghatuverdi, S.K., Gopinath, P.S. y Shanmugan, V. (1987). Controlled study of alexithymic characteristics in patients with psychogenic pain disorder. Psychotherapy and Psychosomatics, 47, 11-17.

Taylor, G.J., Bagby, R.M., Ryan, D.P.A., Doody, K.F. y Keefe, P. (1988). Criterion validity of the Toronto Alexithymia Scale. Psychosomatic Medicine, 25, 500-509. Taylor, G.J., Parker, J.D. y Bagby, R.M. (1990). A preliminary investigation of Alexithymia in men with psychoactive substance dependence. American Journal of Psychiatry, 147, 1228-1230.

Taylor, G.J., Parker, J.D., Bagby, R. M. y Bourke, M. P. (1996). Relationship between Alexithymia and psychological characteristics associated with eating disorders. Journal of Psychosomatic research, 41, 561-568.

Vandereycken, W. y Meerman, R. (1984). Anorexia Nervosa: A Clinican's Guide to Treatment. Berlin: Walter de Gruyter.

Vitousek, K.B. y Orimoto, L. (1993). Cognitive Behavioral Models of Anorexia Nervosa and Obesity. Psychopathology and Gognition. New York: Academic Press. 